Original Article

\title{
EMG activity of the serratus anterior and trapezius muscles during the different phases of the push-up plus exercise on different support surfaces and different hand positions
}

\author{
George Gioftsos, $\mathrm{PhD}^{1)^{*}}$, Michail Arvanitidis, BSc${ }^{1)}$, Dimitrios Tsimouris, BScle, \\ Assimakis Kanellopoulos, $\mathrm{PhD}^{1)}$, George Paras, PhD ${ }^{1)}$, Panagiotis Trigkas, $\mathrm{PhD}^{1)}$, \\ Vasiliki Sakellari, $\mathrm{PhD}^{1)}$ \\ 1) Department of Physiotherapy, School of Health and Caring Professions, Technological Educational \\ Institute (TEI) of Sterea Ellada: $3 \mathrm{rd} \mathrm{km}$ Old National Road Lamia-Athens, 35100 Lamia, Greece
}

\begin{abstract}
Purpose] The appropriate exercise prescription is crucial for achieving scapular stability and providing successful rehabilitation, and the Push-up Plus (PuP) exercise has an important role in shoulder rehabilitation. Consequently, this study examined the effect of support surface stability, hand positioning, and phase of exercise, on the trapezius and serratus anterior muscle contractions as well as on the EMG ratio of the upper/lower trapezius. [Subjects and Methods] Thirteen healthy male volunteers participated in this study. The subjects performed the PuP exercise on stable and unstable supporting surfaces with three different hand orientations. During the PuP exercise, the muscle activities of the upper (UT) and lower (LT) trapezius, as well as the serratus anterior (SA) were measured and expressed as percentages of maximum voluntary isometric contraction (\%MVIC). [Results] The EMG activities of UT and LT were statistically greater during the push-up phase compared to the plus phase of the exercise. The contrary was recorded for the activity of the SA. SA was affected by the support surface as well as by the hand positioning. [Conclusion] The results suggest that different phases of the PuP exercise require different muscle stability actions with corresponding activations of appropriate muscle fibers. A detailed prescription of the required phase of the exercise can more effectively activate the scapula-thoracic musculature.

Key words: Electromyography, Push-up plus, Scapular stabilizers
\end{abstract}

(This article was submitted Feb. 17, 2016, and was accepted Apr. 7, 2016)

\section{INTRODUCTION}

Human ability to independently move the upper extremity is the result of the combined movement of many musculoskeletal structures and of the increased freedom of movement allowed by the necessary decreased stability ${ }^{1,2)}$. The shoulder girdle is the main link of the upper extremity to the trunk, and it plays an important role in the movement quality, while providing anatomical and functional cooperation of the shoulder and scapula-thoracic joints as necessary.

Scapular upward rotation is an essential component of any lifting of the upper extremity, in combination with humeral elevation at the glenohumeral joint. Requisite scapular control and upward rotation are simultaneously obtained by muscle activation patterns. These involve force couples formed by the Upper (UT) and Lower (LT) Trapezius with the Serratus Anterior (SA) muscles. The result of these combined contractions is upward scapular rotation without any displacement of the scapula in the superior or inferior directions due to cooperation of the UT and LT muscles. At the same time, combined action of the whole trapezius muscle and the SA prevents any displacement of the scapula in the side-to-side direction ${ }^{3,4)}$. As a

*Corresponding author. George Gioftsos (E-mail: gioftsos@teilam.gr)

(C2016 The Society of Physical Therapy Science. Published by IPEC Inc.

This is an open-access article distributed under the terms of the Creative Commons Attribution Non-Commercial No Derivatives (by-nc-nd) License $<$ http://creativecommons.org/licenses/by-nc-nd/4.0/>. 
result, a variety of dysfunctions with pathological or clinical symptoms might develop and need to be assessed and addressed by therapists ${ }^{1,5)}$. Consequently, rehabilitation protocols aim for the activation or improvement of muscular cooperation, balance, proprioception, power and strength of the main muscle stabilizers of the scapula ${ }^{3,5)}$.

Exercises contributing to the achievement of therapeutic targets are closed kinetic, and they force the whole upper extremity to function as a unit and in a more harmonic manner during daily life ${ }^{6)}$. These exercises are recognized as therapeutic tools and have great variety and many levels of difficulty during their performance ${ }^{6,7)}$. One of the exercises that is used by therapists, and thus, is an object of study, is the Push-up Plus (PuP). In the PuP exercise, the subject's palms and toes are the supporting parts of the body. The rest of the body forms a straight line from the head to the ankles and with the torso lying near the floor but without touching it. From this starting position, where the elbows are almost in the fully flexed position, the subject raises the body upwards as far as possible by extending the elbows and protracting the scapulae. Thus, PuP includes two phases: (1) the Push-up (Pu) phase that involves the first part of the PuP which ends at the point of completion of elbow extension without scapular protraction, and (2) the Plus (P) phase that is the remaining part of the PuP which starts with completion of elbow movement and continues until the end of the scapular protraction.

$\mathrm{PuP}$ is performed in a closed kinetic chain presupposing muscle cooperation without requiring special equipment. It elicits high activation of the trapezius and serratus anterior muscles which varies even with small performance alterations ${ }^{7)}$. Characteristically, during the PuP exercise, muscle activation and force response increase significantly with increasing levels of exercise difficulty within safe limits ${ }^{3,7)}$.

Different studies have examined the effect of single factors, such as the support surface ${ }^{7,9)}$ or the hand orientation on muscular activity during the $\mathrm{PuP}^{10-13)}$. Due to the complexity of the exercise performance, it is a closed-kinetic activity of the whole body, the effect of different parameters acting simultaneously on the muscle activity must be investigated. Consequently, this study examined the effect of stable and unstable support surfaces, using three different hand positions, on the activities of the trapezius and serratus anterior muscles during the different phases of the push-up plus exercise (Pu and $\mathrm{P})$.

\section{SUBJECTS AND METHODS}

Thirteen healthy male right-handed subjects who led a sedentary life (aged $20.5 \pm 1.0$ years, body weight $79.2 \pm 12.3$ Kgr and height $178.8 \pm 7.2 \mathrm{~cm}$ ) participated in this study. The tested population was recruited from the Department of Physiotherapy through requests for volunteers and represented a population of young students. None of the subjects were professional athletes or were exercising more than five hours per week. The selection criteria for the sample were to be healthy without a history of neck, back or shoulder pain, injury or operation in these areas (determined by a self-completed questionnaire). The study was conducted in accordance with the principles of the Declaration of Helsinki and approved by the Department of Physiotherapy Human Ethics Committee (approval number 124/2013). Confidentiality and anonymity of the participants were maintained at all times, and written informed consent was obtained from all the participants prior to their participation.

The experimental set-up was developed at the Human Performance \& Rehabilitation Laboratory of the Physiotherapy Department where the whole procedure was carried out. Data were collected from the dominant arm and all measurements were recorded in a single session. Initially, the subjects were familiarized with the experimental set-up and the protocol while all exercises were completed in a slow, controlled manner with the aid of a metronome set at a tempo of 60 beats $/ \mathrm{min}$.

Activities of the UT, LT and SA muscles were recorded using surface electromyography. A laboratory system, the dataLINK (type NOSDLK900 with software version 7.5, Biometrics Ltd, UK), was used. Three active EMG sensors SX230 and a twin axis goniometer (Biometrics Ltd) were employed for the measurements. EMG electrodes were placed on the appropriate locations, using double-sided die-cut tapes provided by the manufacturer, in order to record the activities of the UT, LT and SA muscles according to the recommendations of SENIAM ${ }^{14}$. A goniometer was placed on the elbow joint following the manufacturer's guidelines to record the motion of the joint at the same time as the EMG recording. A custom-made stop switch was used to determine the end of the exercise. This was triggered when the maximum distance from the floor was reached by the posterior part of the subject's thoracic spine. All EMG signals were amplified and sampled at 1,000 Hz.

Before testing, anthropometric characteristics were measured and a standardized warm-up procedure ${ }^{13)}$ was used including movement of both arms, such as circumduction and oscillation in the sagittal plane, as well as stretching exercises of the rotator muscles. Subjects then adopted the push-up position by supporting their weight on both hands and feet. The distance between both hands was equal to $120 \%$ of the distance between both the acromions, and the hands were placed at the level of the shoulder joints. The distance between the hands and feet was equal to that measured in the upright position with the shoulders flexed at $90^{\circ}$.

The measured task was performed on a surface that was parallel to the floor. The hands and feet were always in contact with the support surface while the body formed a straight line from the head to the ankles. The subjects performed the PuP exercise by placing their hands on a stable wooden surface or on an unstable one that was constructed by adding a wobble board (rocking side-to-side at a maximum of $16^{\circ}$ ) under the stable wooden surface. They had to balance on those surfaces and not to rock side-to-side during the measured exercise. The feet of the participants were always placed on a stable wooden surface adjusted to the level of the surface used for the hand placement.

Subjects were also asked to perform the exercise with the upper limbs in three different orientations, as follows: 
- Position 1 (P1): the index fingers were placed parallel to each other on the support surface and each arm was allowed to adopt a self-selected position;

- Position 2 (P2): the index fingers were placed parallel to each other on the support surface while each arm was kept in contact with the trunk of the subject; and

- Position 3 (P3): the index fingers were placed at $45^{\circ}$ of internal rotation compared to P1 and each arm was kept at $90^{\circ}$ of abduction at the shoulder joint.

Participants were asked to perform 3 successful repetitions of the tested trials while a rest period of 1 min was allowed between repetitions to avoid fatigue. The order of the performance of the conditions was randomly allocated. Each volunteer performed 18 successful repetitions of PuP exercises, that is: [3 trials] $\times[2$ surfaces (stable vs unstable) $] \times[3$ hand positions (P1, P2 \& P3)]. Finally, three successful trials of the Maximum Voluntary Isometric Contraction (MVIC) of each measured muscle were recorded following a muscle testing procedure suggested by previous research ${ }^{15}$.

During data collection, the raw EMG recordings were the monitored, full-wave rectified and processed using a root mean square (RMS) algorithm with a 3-ms moving window. Measurements were expressed in millivolts and the mean value of the three trials for each muscle was used for the estimation of the activation of each tested muscle. Finally, the EMG activity values of each measured muscle during the PuP exercise were expressed as a percentage of the EMG values produced by the muscle during the muscle testing performance, \% MVIC.

The signal of the goniometer was used to monitor the start and the end of the elbow movement and at the same time to mark the start and the end of the push-up phase of the PuP exercise. The signal of the back-stop was used to monitor the end of the PuP exercise. Consequently, those two signals were the discriminating landmarks of the two phases of the measured $\mathrm{PuP}$ exercise, that is the $\mathrm{Pu}$ and the $\mathrm{P}$ phase.

The intraclass correlation coefficient (ICC) was used to determine the same day test-retest reliability of the three EMG recordings. In order to determine if there were significant differences in the EMG activities of UT, LT, SA, as well as in the UT/LT activity rations among the different phases of the exercise, hand positioning and support surfaces, analysis of variance (ANOVA) was performed. A least squares difference (LSD) pairwise multiple comparison analysis was performed to determine the significance of the differences between pairs of means. Statistical analysis was performed using SPSS 20.00 software and significance was accepted in all statistical tests for values of $\mathrm{p}<0.05$.

\section{RESULTS}

The mean value $( \pm \mathrm{SD}$ ) of EMG activities the three trials of the muscle testing, with the ICC values between these recordings for each tested muscle, were as follows: UT, $1.78 \pm 0.21 \mathrm{mV}(\mathrm{ICC}=0.80-0.87)$; LT, $1.75 \pm 0.36 \mathrm{mV}(\mathrm{ICC}=0.96-0.97)$ and UT, $1.38 \pm 0.5 \mathrm{mV}(\mathrm{ICC}=0.98-0.98)$.

The overall activation of the UT showed statistically significant difference with regard to the phase of the exercise ( $p<0.05$ and $\mathrm{F}=8.3$ ) and the position of the hands $(\mathrm{p}<0.05$ and $\mathrm{F}=4.7)$, but it was not affected by the stability of the support surface $(\mathrm{p}>0.05$ and $\mathrm{F}=1.2)$. More specifically, the differences between the Pu phase $(30.2 \pm 13.6 \% \mathrm{MVIC})$ and $\mathrm{P}$ phase $(24.1 \pm$ $8.5 \% \mathrm{MVIC})$, as well as between the hand positions of P1 (25.1 $\pm 8.8 \% \mathrm{MVIC})$ and P2 (29.5 $\pm 12.6 \% \mathrm{MVIC})$ were statistically significant. Similar to the UT, the overall activation of LT was also significantly altered by the phase of the exercise ( $<<0.05$ and $\mathrm{F}=7.1)$ and the position of the hands $(\mathrm{p}<0.05$ and $\mathrm{F}=5.2)$. The $\mathrm{P}$ phase $(4.1 \pm 3.8 \% \mathrm{MVIC})$ was significantly different from the Pu phase $(9.8 \pm 11.1 \% \mathrm{MVIC})$, while hand position P1 (6.6 $\pm \% \mathrm{MVIC})$ was significantly different from hand position $\mathrm{P} 2$ $(8.7 \pm \%$ MVIC).

The overall activation of the SA showed statistically significant differences with regard to all tested parameters, such as exercise phase $(\mathrm{p}<0.001$ and $\mathrm{F}=72.9)$, position of the hands $(\mathrm{p}<0.05$ and $\mathrm{F}=3.9)$, and the stable or unstable support surface $(\mathrm{p}>0.05$ and $\mathrm{F}=6.4)$. More specifically, the differences between the Pu phase $(49.2 \pm 17.9 \% \mathrm{MVIC})$ and $\mathrm{P}$ phase $(75.9 \pm$ $16.1 \% \mathrm{MVIC})$ as well as between the hand positions of P2 (59.6 $\pm 17.6 \% \mathrm{MVIC})$ and P3 (66.3 $\pm 22.3 \% \mathrm{MVIC})$, and finally between the stable $(65.9 \pm 21.8 \% \mathrm{MVIC})$ and unstable $(59.9 \pm 17.3 \% \mathrm{MVIC})$ support surfaces, were statistically significant.

Finally, the UT/LT activity ratio was significantly affected by the phase of the exercise $(\mathrm{p}<0.05$ and $F=5.7)$, and the $P$ phase $(8.1 \pm 4.3)$ was significantly different from the Pu phase $(6.0 \pm 4.5)$. Table 1 shows the EMG activities $(m e a n \pm S D)$ of the measured muscles (UT, LT \& SA) under the tested experimental conditions with the statistical significance of the differences.

\section{DISCUSSION}

The PuP exercise is an important tool for therapists, aiming to deal with problems of the upper extremity and specifically to improve the stability of the scapula-thoracic joint by increasing muscular cooperation ${ }^{1,13}$ ). Such a demanding task requires well-documented supervision and guidance during its performance resulting from the extensive study of the effect of different parameters on muscle activation ${ }^{8)}$. So, the study of the muscle performance, during PuP and the simultaneous effect of different conditions to overcome them can be crucial. In the present study an effort was made to identify the effects elicited by the support surface, hand positions, and phase of the PuP exercise.

Different phases of the exercise require different muscle activation with a variety of activation levels, due to different movements taking place and the different ranges of movement used ${ }^{8)}$. The Pu phase mainly involves elevation of the upper 
Table 1. The EMG activities (mean values \pm SD) of the measured muscles (UT, LT and SA) as a percent of maximum voluntary isometric contraction (\%MVIC) and the UT/LT ratio during the phases of the push-up plus exercise (Push-up and Plus) on stable and unstable supporting surfaces with three different hand positions (P1, P2 and P3)

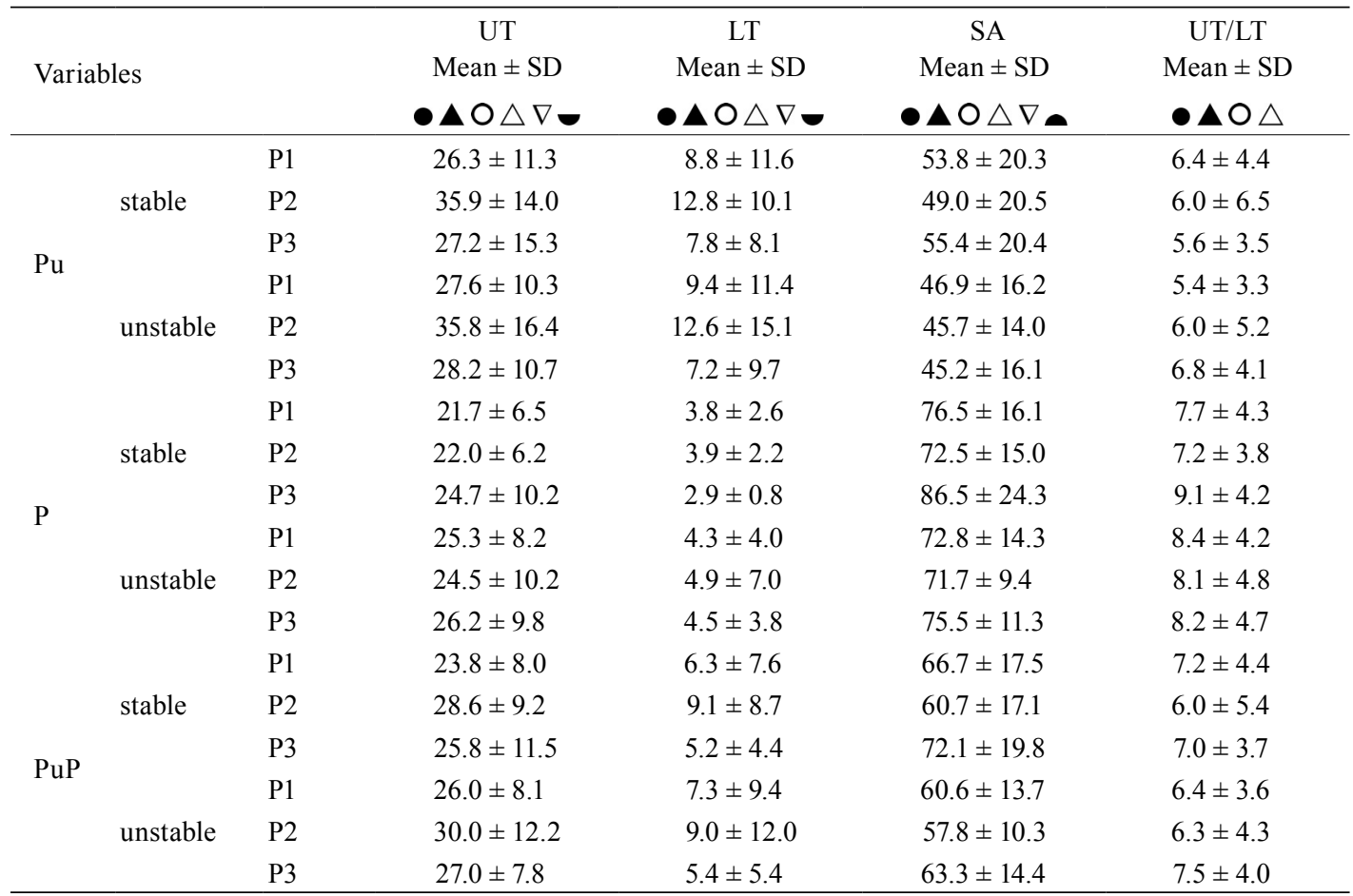

-: The phase of movement showed a statistically significant difference $(\mathrm{p}<0.05)$

$\boldsymbol{\Delta}$ : The hand position showed a statistically significant difference $(\mathrm{p}<0.05)$

- The supporting surface showed a statistically significant difference $(\mathrm{p}<0.05)$

$\mathrm{O}$ : $\mathrm{P}$ and $\mathrm{Pu}$ showed a statistically significant difference $(\mathrm{p}<0.05)$ and $(\mathrm{p}<0.001$ for SA)

$\triangle: \mathrm{P}$ and $\mathrm{PuP}$ showed a statistically significant difference $(\mathrm{p}<0.05)$ and $(\mathrm{p}<0.001$ for $\mathrm{SA})$

$\nabla: \mathrm{Pu}$ and $\mathrm{PuP}$ showed a statistically significant difference $(\mathrm{p}<0.05)$ and $(\mathrm{p}<0.001$ for SA)

-: P1 and P2 showed a statistically significant difference $(\mathrm{p}<0.05)$

-. P2 and P3 showed a statistically significant difference $(\mathrm{p}<0.05)$

Pu: push-up; P: plus; PuP: push-up plus; P1, P2 \& P3, the three positions of the hand; UT: upper trapezius; LT: lower trapezius; SA: serratus anterior

arm with associated movement of the scapula resulting from the activation of the UT and LT. In contrast, the P phase only involves movement of the scapula leading to activation mainly of the SA. At the same time, cooperation of UT and LT expressed by the UT/LT ratio, increases. This muscle cooperation can be explained by the present findings which are in agreement with the results of similar studies ${ }^{4,6}$. Statistically significant differences were recorded for UT, LT and the UT/LT ratio during the $\mathrm{Pu}$ phase and for SA during the $\mathrm{P}$ phase.

The stability of the supporting surface alters the stability of the human body and it is an important factor demanding different patterns of muscular cooperation ${ }^{5,6}$. Consequently, stable and unstable supports were used in an attempt to identify their effects on muscular activity and the resulting movement patterns. When subjects tried to avoid rocking side-to-side on the unstable surface, they introduced abduction-adduction to the shoulder joints leading to abduction-adduction of the scapula, altering the muscle involvement. The present results suggest that an unstable support surface only has a significant effect on the activity of SA, with the stable surface increasing SA muscular activity. These findings can be explained by the fact that abduction-adduction of the scapula is involved; therefor the SA must be activated. Why is the SA activity on an unstable supporting surface less than that on a stable one? One possible explanation is that there is a decrease in the Pu phase of the task resulting from a fear of falling or the mobilization of other muscles such as the horizontal adductors of the shoulder. $\mathrm{PuP}$ is a demanding task that requires activation of the whole body and is difficult to supervise and consequently perform properly. A possible solution to this problem might be the use of optoelectronic techniques to record movement in 3D. Not surprisingly, our present findings are in conflict with previously published results ${ }^{7}$. PuP on unstable surfaces has been found 
to increase SA muscle activity ${ }^{4,7,9)}$, or SA in combination with UT, and to increase the activity of the trapezius while decrease the activity of $\mathrm{SA}^{16}$ ). Considering that the unstable surface had a low level of instability (rocking of $16^{\circ}$ ), and only in one direction (side-to-side), the effect of instability on muscular activity needs to be investigated further.

The hand position in Pup changes the degree of arm elevation in the frontal plane, altering the range of arm movement as well as the movement itsself ${ }^{10-12}$. Flexion, abduction or horizontal abduction is required at the shoulder joint, leading respectively, to upward rotation with retraction, or adduction of the scapula. UT and LT demonstrated the same activity patterns in response to hand positioning, and axially showed statistically significant increased involvement in hand position $\mathrm{P} 2$. As this position elicits shoulder flexion during the PuP, and consequently upward rotation and protraction of the scapular, increased movement and stability of the scapula was needed in the frontal plane. In contrast, SA showed significant increase in activity in hand position P3, that is when scapular adduction occurred, replacing scapular movement in the frontal plane. Nevertheless, scapular stability in this plane wasn't necessary.

The present findings suggest that different phases of the PuP exercise require different muscle activities and co-operation. Similarly, the type of supporting surface and the positioning of the hands affect muscle activities in a manner that is dependent on the phase of the exercise making its prescription a highly demanding therapeutic task.

The present study had some limitations. The data was obtained from a small group of young, healthy male adults and only their dominant right hand was measured. Therefore, the extrapolation of the findings to patients or to the general population should be performed with caution.

\section{ACKNOWLEDGEMENT}

The authors wish to thank Katie Somerscales for her help in proofreading the manuscript.

\section{REFERENCES}

1) Kibler WB, McMullen J: Scapular dyskinesis and its relation to shoulder pain. J Am Acad Orthop Surg, 2003, 11: 142-151. [Medline] [CrossRef]

2) Neumann DA: Kinesiology of the musculoskeletal system: foundation for physical rehabilitation. St. Louis: Mosby, 2002, pp 123-144.

3) Yoo WG: Effect of exercise speed and isokinetic feedback on the Middle and Lower Serratus Anterior muscles during push-up exercises. J Phys Ther Sci, 2014, 26: 645-646. [Medline] [CrossRef]

4) Ekstrom RA, Donatelli RA, Soderberg GL: Surface electromyographic analysis of exercises for the trapezius and serratus anterior muscles. J Orthop Sports Phys Ther, 2003, 33: 247-258. [Medline] [CrossRef]

5) Seo SH, Jeon IH, Cho YH, et al.: Surface EMG during push-up plus exercise on a stable support or swiss ball: scapular stabilizer muscle exercise. J Phys Ther Sci, 2013, 25: 833-837. [Medline] [CrossRef]

6) McMullen J, Uh1 TL: A kinetic chain approach for shoulder rehabilitation. J Athl Train, 2000, 35: 329-337. [Medline]

7) Ludewig PM, Hoff MS, Osowski EE, et al.: Relative balance of serratus anterior and upper trapezius muscle activity during push-up exercises. Am J Sports Med, 2004, 32: 484-493. [Medline] [CrossRef]

8) Park SY, Yoo WG: Differential activation of parts of the serratus anterior muscle during push-up variations on stable and unstable bases of support. J Electromyogr Kinesiol, 2011, 21: 861-867. [Medline] [CrossRef]

9) Kim SH, Kwon OY, Kim SJ, et al.: Serratus anterior muscle activation during knee push-up plus exercise performed on static stable, static unstable, and oscillating unstable surfaces in healthy subjects. Phys Ther Sport, 2014, 15: 20-25. [Medline] [CrossRef]

10) Lee SY, Lee DH, Park JS: The effects of changes in hand position on the electromyographic activities of the shoulder stabilizer muscles during push-up plus exercises on unstable surfaces. J Phys Ther Sci, 2013, 25: 125-128. [CrossRef]

11) Cogley RM, Archambault TA, Fibeger JF, et al.: Comparison of muscle activation using various hand positions during the push-up exercise. J Strength Cond Res, 2005, 19: 628-633. [Medline]

12) Cho SH, Baek IH, Cheon JY, et al.: Effect of the push-up plus (PUP) exercise at different shoulder rotation angles on shoulder muscle activities. J Phys Ther Sci, 2014, 26: 1737-1740. [Medline] [CrossRef]

13) Suprak DN, Bohannon J, Morales G, et al.: Scapular kinematics and shoulder elevation in a traditional push-up. J Athl Train, 2013, 48: 826-835. [Medline] [CrossRef]

14) Hermens HJ, Freriks B, Disselhorst-Klug C, et al.: Development of recommendations for SEMG sensors and sensor placement procedures. J Electromyogr Kinesiol, 2000, 10: 361-374. [Medline] [CrossRef]

15) Ekstrom RA, Soderberg GL, Donatelli RA: Normalization procedures using maximum voluntary isometric contractions for the serratus anterior and trapezius muscles during surface EMG analysis. J Electromyogr Kinesiol, 2005, 15: 418-428. [Medline] [CrossRef]

16) Pirauá AL, Pitangui AC, Silva JP, et al.: Electromyographic analysis of the serratus anterior and trapezius muscles during push-ups on stable and unstable bases in subjects with scapular dyskinesis. J Electromyogr Kinesiol, 2014, 24: 675-681. [Medline] [CrossRef] 